\title{
The Effect of Age in the Shape of the Spermatozoa Frozen Friesian Bulls
}

\author{
Makki Khalaf Hussain Al-Dulaimi \\ Department of Animal Production Techniques, Technical College Musayab, Al-Furat Technical \\ University, Babylon, Iraq. \\ Makkikhalaf@yahoo.com
}

Submission date:- 18/4/2018 Acceptance date:- 26/3/2018 Publication date:- 16/10/2018

Keywords: bull sperm, morphology of head sperm, dimension of head sperm.

\begin{abstract}
This study was aimed to determine whether there are any differences in the shape and dimensions of bulls sperm using different ages, as well as to know the maturity of the sperm, then choose the bull for insemination or to freeze the taken semen to be used in artificial insemination. Now a day become morphology shape for sperm important item in valuation of the semen and chose the best normal sperm whence dimension and shape. Methods were used and bulls were classified by age into three groups, the first group includes 4 bulls aged 2-4 years, second group includes 4 bulls 4-6 years old, third group includes 4 bulls 6-8 years old. Semen of each group was taken randomly and the mature and intact semen were examined. The results were revealed that the highest value of head's length (6-8 years category) was (8.20), while for 4-2 years category was the least (7.81). For the width, the third category was recorded the highest (4.51) along with the other. The Eillipticity inducts that 4-2 years group was the highest value (1.85), while for elongation character the 2-4 years group was the highest with $(0.3)$ compared with the other.
\end{abstract}

It can be concluded our study in the current to the next score whenever the bull offers in the form of life has become the best sperm beltala can choose sperm in the freezer.

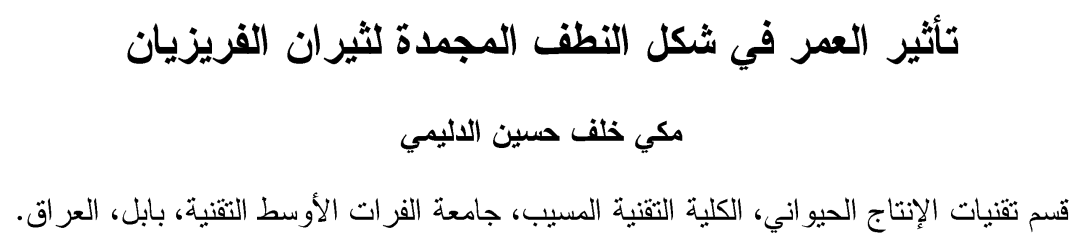

Makkikhalaf@yahoo.com 


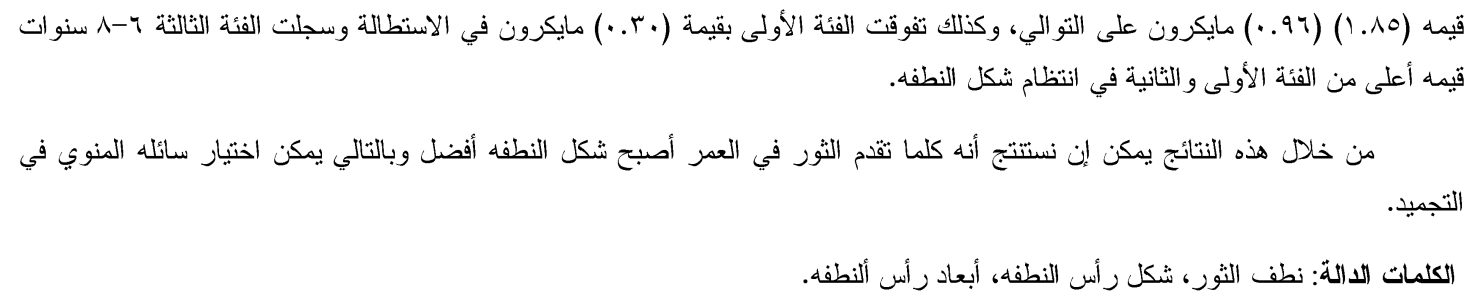

يعاني النشاط التناسلي في شتى أنحاء العالم من مشاكل عديدة منها مشاكل بيئية ومشاكل ور اثية. وتعد إدخال التقتيات ألحديثه و ولوم

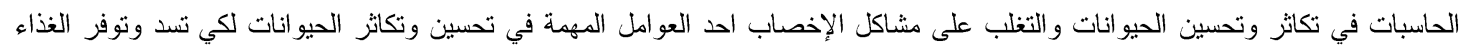

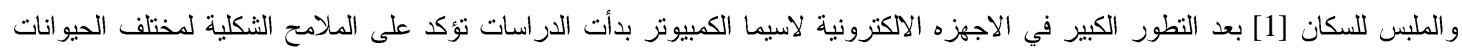

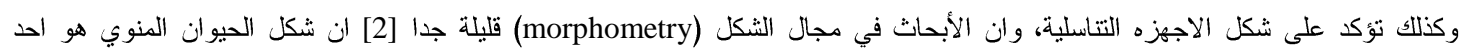

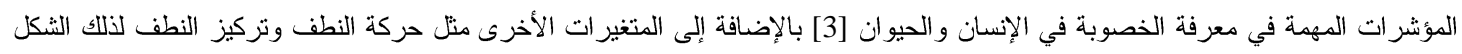
غير الطبيعي للنطفة يفقدها القدرة على الإخصاب حتى إذا كانت الحركة والتركيز والكثافة طبيعية. إن السباحة التقدية للنطف تدل على التى أنها تمنالكي رأسا سليما شكلياً لان النطف ذات الرأس المنظظم يكون ذيلها منتظم أيضا [4] لذلك حظيت التباينات في الحيو انات المنوية من ولنئ حيث الإبعاد

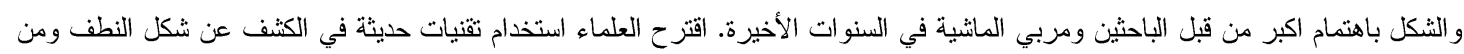

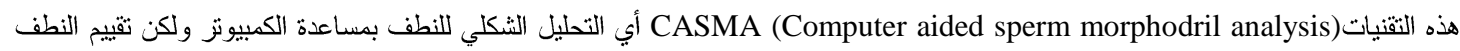

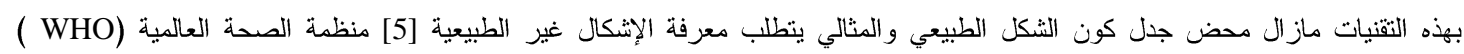

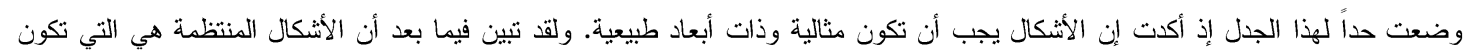

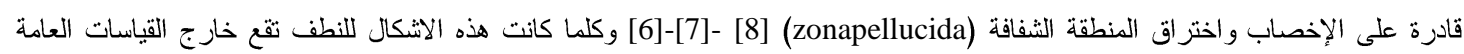

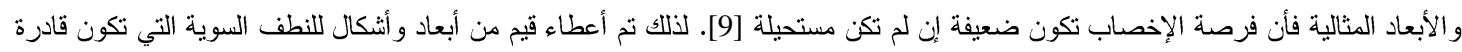

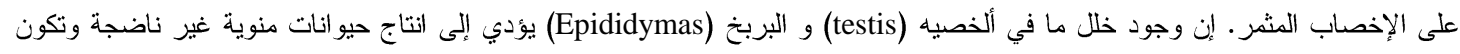

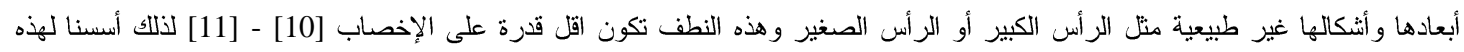

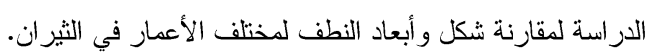

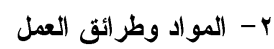

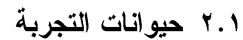

جُلب السائل المنوي من محطة ابقار الفيحاء - ناحية مشروع المسيب - قضاء المحاويل - محافظة بابل بو اقع 30 قصبة ل كا ثثور 10

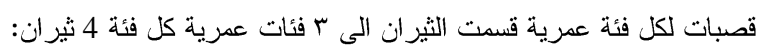
الفئة الاولى 2-4 سنة الفئة الثانية 4-6 سنة الفئة الثالثة 6-8سنة جمع العينات r.r

أحظرت عينات التجربة إلى مختبر فسلجة التتاسل التابع لقسم تقنيات الإنتاج الحيواني في الكلية التقنية المسيب/ جامعة الفرات الأوسط

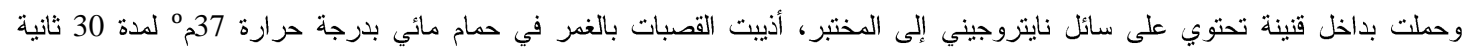
ومزجت بصبغة الايوسين- نيكروسين.

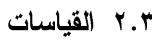

قيس طول راس ألنطفه بواسطة (الكاميرا المجهر) المرتبطة بالحاسوب وذلك من منطقة القلنسوه (Acrosome) إلى بداية القطعة الوسطية صورة (r). وقيس عرض النطفة وذلك من اعرض نقطة في رأس النطفه صورة (1) أما مساحة الرأس فقيست بحسب القانون التالي

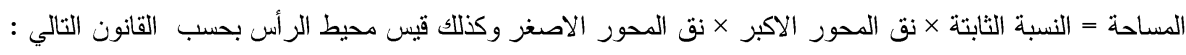




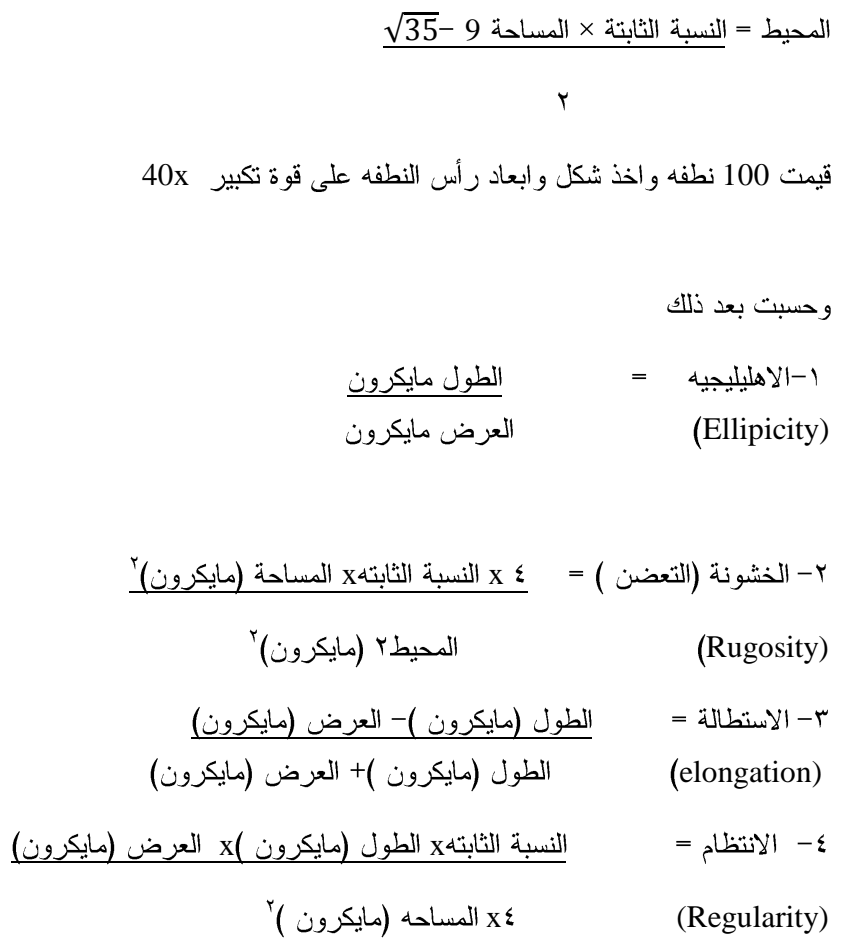

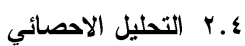

استعمل التصميم العشو ائي الكامل (CRD) لدراسة تأثير العمر على الصفات المختلفة على شكل وأبعاد رأس ألنطفه وقورنت الفروق

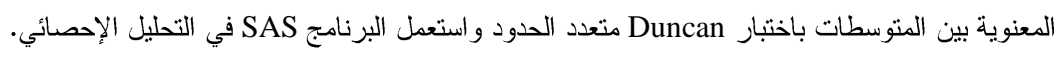

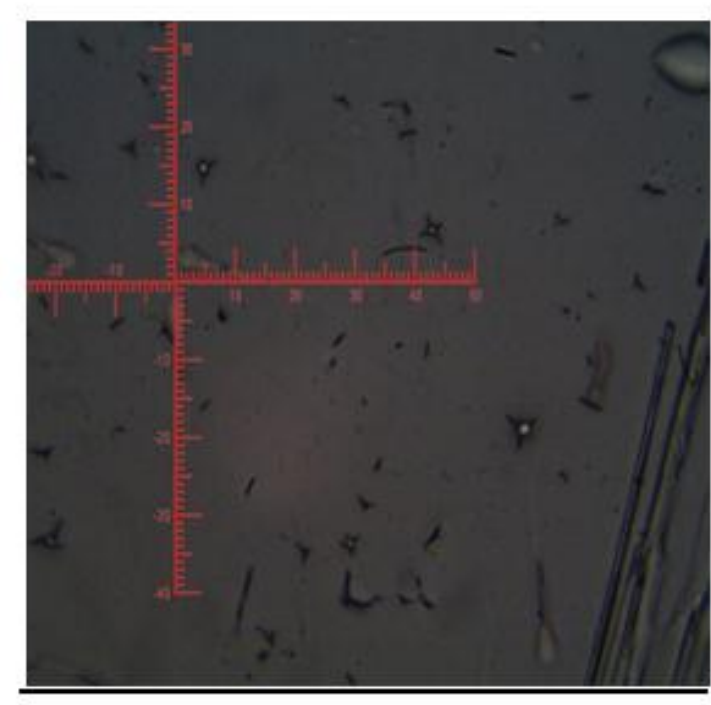

صورة (ץ) قياس طول رأس ألنطفه

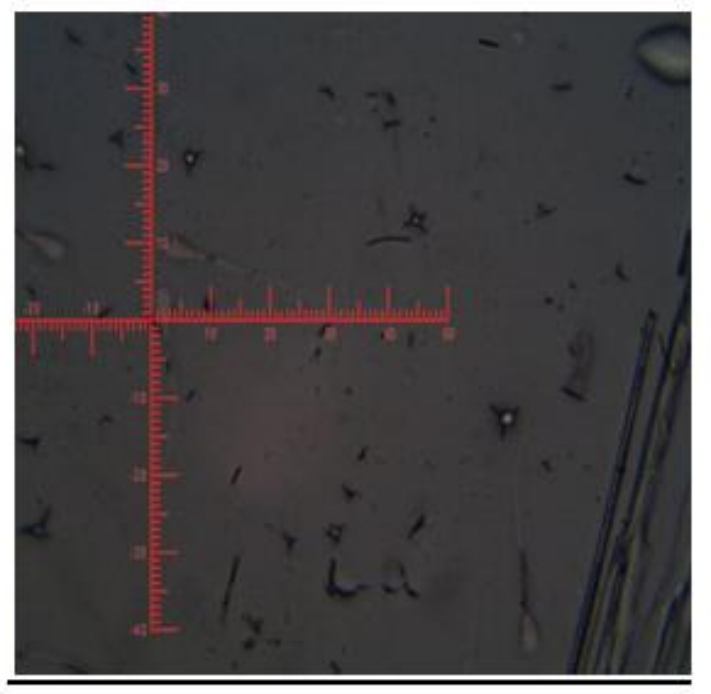

صورة (1) قياس عرض رأس ألنطفه

النتائج المستحصلة من الفئات العمرية (الفئة الأولى 2-4 سنة، الفئة الثانية 4-6 سنة والفئة الثالثة 6-8سنة) وبعد التحليل الإحصائي

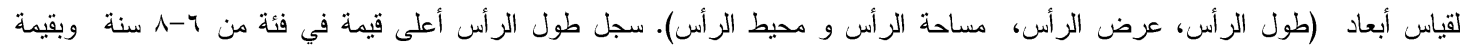

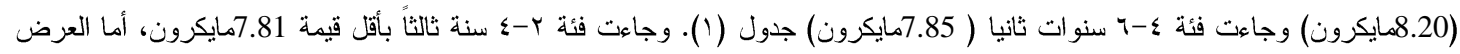

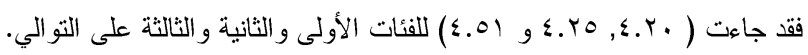


أما بالنسبة لمساحة الرأس كانت الفئة الثالثة متفوقة ( 29.50) مايكرون` وسجلت الفئة الأولى اقل في المساحة (28.03) مايكرونج وقد

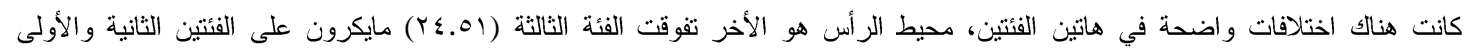

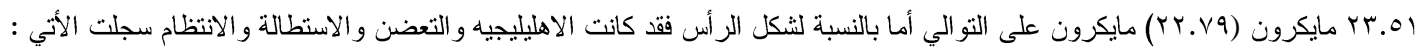
• الاهليليجية سجلت الفئة الأولى أعلى قيمة من باقي الفئات (1.85) بينما الفئة الثالثة هي الأقل (1.81) إن تفوق الفئة الأولى يعني إن الثكل

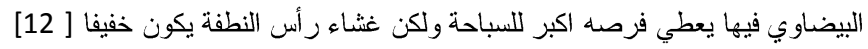

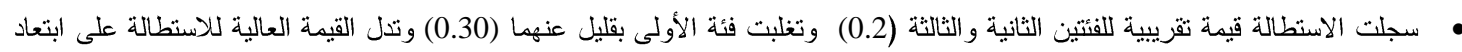
النطف عن الثنكل المثالي. [13]

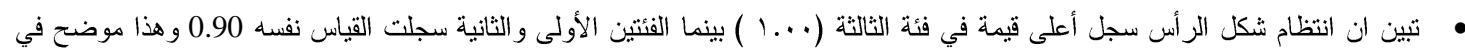
الجدول (r). (1). • الخشونه (التعضن) سجلت الفئة الأولى (19. . ) وكانت هي الأعلى أما الفئة الثالثة فكانت هي الأقل (10. . ) أن القيمة الو اطئة تعني أن النطفة

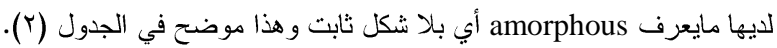

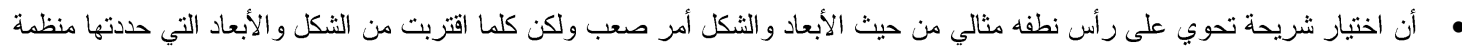

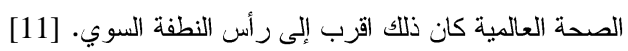

جدول (1) يبين أبعاد رأس النطقة

\begin{tabular}{|c|c|c|c|c|}
\hline \multirow[t]{2}{*}{ المتوسط العام } & \multicolumn{3}{|c|}{ الفئات العمرية } & \multirow[t]{2}{*}{ الصفات } \\
\hline & 6-6 سنة & 6-6 سنة & 2-4سنة & \\
\hline $7.95 \pm 0.30$ & $\begin{array}{c}8.20 \pm 40.21 \\
\mathrm{a}\end{array}$ & $\begin{array}{c}7.85 \pm 0.38 \\
b\end{array}$ & $\begin{array}{c}7.81 \pm 0.33 \\
\mathrm{c}\end{array}$ & الطول (مايكرون) \\
\hline $4.32 \pm 0.15$ & $\begin{array}{c}4.51 \pm 0.19 \\
\mathrm{a}\end{array}$ & $\begin{array}{c}4.25 \pm 0.16 \\
b\end{array}$ & $\begin{array}{c}4.20 \pm 0.12 \\
\mathrm{c}\end{array}$ & العرض(مايكرون) \\
\hline $28.75 \pm 0.3$ & $\begin{array}{c}29.50 \pm 0.25 \\
\mathrm{a} \\
\end{array}$ & $\begin{array}{c}28.20 \pm 0.20 \\
b\end{array}$ & $\begin{array}{c}28.18 \pm 0.45 \\
b\end{array}$ & المساحة (مايكرون)' \\
\hline $23.34 \pm 0.26$ & $\begin{array}{c}24.51 \pm 0.26 \\
\mathrm{a}\end{array}$ & $\begin{array}{c}23.10 \pm 0.27 \\
b\end{array}$ & $\begin{array}{c}22.79 \pm 0.25 \\
c\end{array}$ & المحيط (مايكرون)' \\
\hline
\end{tabular}

المتوسطات التي تحمل حروفا مختلفة ضمن الصف الو احد تختلف معنويا فيما بينها (P<0.05)

قام [14] بدر اسة شكل وحجم الحيوانات المنوية بمساعدة الكمبيوتز وتحليل الحيو انات المنوية المجمدة للكبش وسلط الضوء على دقة

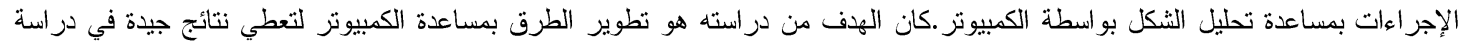

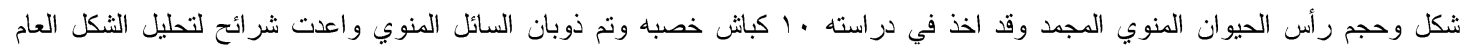

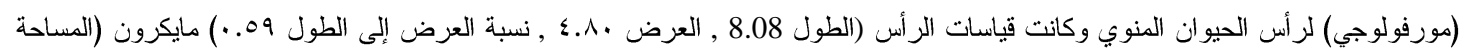

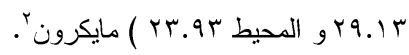

جدول (ץ) يبين الخصائص الثكلية لرأس النطفة

\begin{tabular}{|c|c|c|c|c|}
\hline \multirow[t]{2}{*}{ المتوسط العام } & \multicolumn{3}{|r|}{ الفئات العمرية } & \multirow[t]{2}{*}{ الصفات } \\
\hline & 6-6 سنة & 6-6 سنة & 4-2 سنة & \\
\hline $1.83 \pm 0.06$ & $\begin{array}{c}1.81 \pm 0.08 \\
b\end{array}$ & $\begin{array}{c}1.84 \pm 0.06 \\
\mathrm{a}\end{array}$ & $\begin{array}{c}1.85 \pm 0.05 \\
\mathrm{a}\end{array}$ & الاهليليجية \\
\hline $0.86 \pm 0.04$ & $\begin{array}{l}0.65 \pm 0.06 \\
\mathrm{c}\end{array}$ & $\begin{array}{l}0.72 \pm 0.05 \\
b\end{array}$ & $\begin{array}{c}0.96 \pm 0.03 \\
\mathrm{a}\end{array}$ & الخشونه(التعضن) \\
\hline $0.29 \pm 0.01$ & $\begin{array}{l}0.29 \pm 0.01 \\
\mathrm{a}\end{array}$ & $\begin{array}{l}0.29 \pm 0.01 \\
\mathrm{a}\end{array}$ & $\begin{array}{c}0.30 \pm 0.02 \\
\mathrm{a}\end{array}$ & الاستطالة \\
\hline $0.93 \pm 0.3$ & $\begin{array}{c}1.00 \pm 0.04 \\
\mathrm{a}\end{array}$ & $\begin{array}{c}0.90 \pm 0.03 \\
b\end{array}$ & $\begin{array}{c}0.90 \pm 0.03 \\
b\end{array}$ & الانتظام \\
\hline
\end{tabular}

المتوسطات التي تحمل حروفا مختلفة ضمن الصف الواحد تختلف معنويا فيما بينها (P<0.05) 
بعد الإذابة (thowing) تبين أنه كلما تقدم الثور في العمر أصبح شكل النطفه أفضل وبالتالي يمكن اختيار سائله المنوي في التجميد.

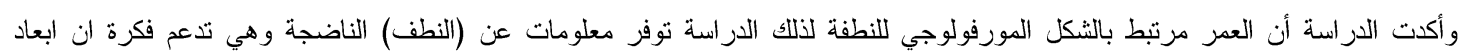

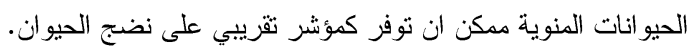

\section{CONFLICT OF INTERESTS}

There are no conflicts of interest.

\section{References}

[1] C. De Geyter, M. De Geyter, B. Koppers, and E. Nieschlag, "Diagnostic accuracy of computer-assisted sperm motion analysis,” Human Reproduction, vol. 13, no. 9, pp. 2512-2520. 1998.

[2] P. De Paz, M. Mata-Campuzano, E.J. Tizado, M. Álvarez, M. Álvarez-Rodríguez, P. Herraez, and L. Anel, "The relationship between ram sperm head morphometry and fertility depends on the procedures of acquisition and analysis used, " Theriogenology. 76, 1313- 1325. 2011.

[3] C. G. Gravance, I. K. M. Liu, R. O. Davis, J. P. Hughes, "Quantification of normal head morphometry of stallion spermatozoa," Journal of Reprod. and Fertility, 108:1,41-46. 1996.

[4] H. A. Abdel-Rahman, M. S. Belely, A. A. Al-Qarawi, "The relationship between semen quality and mineral composition of semen in various ram breed . Small Rumin,”. Res.; 38(1):45-49. 2000.

[5] Al. Dulaimi Makki Khalaf Hussein, Dana Tapaloaga, Paul- Rodian Tapaloaga, Carmen Daniela Petcu, "Results Regarding Some Morphometric Features of Spermatozoa in Ram, Agriculture and Agricultural Science Procedia," Volume 6, , Pages 232- 235, ISSN 2210-7843. 2015.

[6] K. F. Dowsett, and L. M. Knott, "The influence of age and breed on stallion semen," Theriogenology. 46(3), 397-412. 1996

[7] G. H. Coulter, R. B. Cook, and J. P. Kastelic, "Effects of dietary energy on scrotal surface, temperature, seminal quality, and sperm production in 5- 5young beef bulls," J. Anim. Sci. 75(4), 1048-1052. 1997.

[8] N. Cornilă, "Morfologia microscopică a animalelor domestice," 4vol I si II, Ed. Bic All. 2000

[9] S. H Al-Samarrae, Potentiality employment of some hematological and biochemical criterions for evaluation of productivity performance traits of Iraqi sheep,” Ph.D. Thesis, University of Baghdad. 2006.

[10] S. A. Kidd, B. Eskenazi, and A. J. Wyrobek, "Effects of male age on semen quality and fertility: 1A review of the literature," Fertil. Steril. 75(2), 237-248. 2001.

[11] R. Eliasson, "Semen analysis with regard to sperm number, sperm morphology and functional aspects," Asian Journal of Andrology, vol. 12, no. 1, pp. 26-32. 2010.

[12] L. Anel, M. Alvarez, E. Anel, C. Rodriguez, M. Kaabi, J. Boixo, "Effect of pre-freezing dilution rate on quality and fertility of ram thawed semen," ESDAR Newslett, 6: 49- 50.2001.

[13] J. L. Bailey, J. Bilodeau, and N. Cormier, "Semen cryopreservation in domestic animals: A damaging and capacitating phenomenon," J. Androl. 21(1), 1-7. 2000.

[14] Tapaloaga, Dana, "Cercetari de micromorfometrie computerizata asupra spermatozoizilor de vier, Teza de doctorat,” USAMV Bucuresti. 2004. 